\title{
Laboratoire du praticien et sécurité des patients
}

\begin{abstract}
Les laboratoires de cabinet médical sont menacés de disparition par la révision de la liste des analyses effectuée par l'Office fédéral de la santé publique. L'OFSP cherche à faire des économies - et ce sera sur le dos des laboratoires de cabinet et de la sécurité des patients! Selon l'OFSP, la révision prévue entraînera des pertes de revenu de l'ordre de 22 à $25 \%$. Nos calculs, fondés sur des critères d'économie d'entreprise, indiquent des pertes beaucoup plus importantes pouvant atteindre $36 \%$. Il ne sera ainsi plus possible de couvrir les frais d'exploitation d'un laboratoire au cabinet. Chaque médecin devra alors décider s'il veut financer indirectement son labo par d'importants moyens issus d'autres secteurs du cabinet ou confier ses analyses à un laboratoire extérieur.
\end{abstract}

\section{La disparition du laboratoire au cabinet menacerait la sécurité des patients tout en entraînant une baisse de qualité et une perte de confort pour ces derniers}

Quelles seraient les conséquences d'une disparition du laboratoire du praticien? Les médecins de famille ont besoin d'un laboratoire dans leur pratique quotidienne. Cet outil permet d'objectiver les données à disposition et contribue de manière décisive au diagnostic et à l'établissement du traitement. Externaliser les analyses aurait des conséquences radicales: la disparition du laboratoire au cabinet menacerait la sécurité des patients tout en entraînant une baisse de qualité et une perte de confort pour ces derniers. Il en résulterait de longues attentes du diagnostic - résultats seulement le lendemain -, des décisions thérapeutiques retardées, des traitements et hospitalisations inutiles ainsi que des visites médicales supplémentaires. Sans laboratoire au cabinet, il deviendrait impossible d'assurer les soins d'urgence dans les régions retirées. En évaluant le laboratoire du praticien hors contexte, l'OFSP entraîne des économies mal placées, des transferts de coûts et en fin de compte un renchérissement global. Le système performant des cabinets de premier recours sera démantelé et l'on privera le médecin de famille d'un instrument de travail important, réduisant ainsi encore l'attrait de la profession.

La FMH a déjà signalé plusieurs fois que la taxe de présence envisagée par l'OFSP pour compenser les désavantages que subirait le laboratoire du cabinet face au laboratoire mandaté est une absurdité. Forfaitaire, elle ne tient pas compte des grandes différences entre spécialités - pédiatres, médecins de premier recours, divers spécialistes quant au nombre et à l'éventail des analyses effectuées.
En collaboration avec Anton Prantl (Caisse des médecins) et Roman Fried (Centre de contrôle qualité des laboratoires de Zurich) et en se fondant sur le diagnostic en présence du patient, la FMH a développé son propre modèle («Point-of-Care Diagnostic») qui représente les charges du laboratoire du praticien (salaires, coût des appareils et réactifs, etc.) par des calculs économiquement pertinents résultant d'une analyse des processus. L'idée directrice de ce modèle réside dans une tarification séparée des analyses de laboratoire exécutées en présence du patient. On aurait ainsi, par exemple, une position «CRP» et une position «CRP poc». Le 30 octobre 2008, nous avons présenté à l'OFSP le modèle «Point-of-Care Diagnostic» et les problèmes que pose la taxe de présence. La décision définitive de l'OFSP n'est pas encore tombée. Notre solution est neutre quant aux coûts. Il n'en découle pas d'économies potentielles pour l'OFSP, mais pas non plus d'augmentation des coûts.

Nous en appelons au sens des responsabilités de l'OFSP. Avec son projet, le laboratoire de cabinet et le savoir-faire s'y rapportant s'effondreront, la sécurité des patients sera mise en péril et les assistantes médicales ne seront plus formées à la pratique du laboratoire.

Que va-t-il se passer maintenant? Selon un communiqué de l'OFSP, l'introduction de la révision a été repoussée à une date indéterminée. Ne connaissant pas la décision

\section{En évaluant le laboratoire du praticien hors contexte, I'OFSP entraîne des économies mal placées}

de l'OFSP et présumant qu'il insistera sur la taxe de présence et le potentiel d'économies, l'Assemblée des délégués de la FMH a décidé à l'unanimité le 19 novembre 2008 de mettre en œuvre un catalogue de mesures prévoyant des mesures publiques, sous l'égide de la FMH. Développé avec le large appui des milieux concernés (GT Laboratoire et sociétés cantonales et de discipline), ce catalogue comprend plusieurs axes. Des mesures sont prévues tant sur le plan technique, politique et médiatique qu'au niveau de la base. Elles ont pour objectif d'obtenir une réorientation complète de la révision de la liste des analyses et seront mises en œuvre avec l'ensemble du corps médical, selon un calendrier et des priorités définis. «L'union fait la force».

Dr Ernst Gähler, vice-président de la FMH, responsable du domaine Tarifs et conventions 